EPJ Web of Conferences 84, 03001 (2015)

DOI: $10.1051 /$ epjconf/20158403001

(C) Owned by the authors, published by EDP Sciences, 2015

\title{
Studies of HeH: DR, RIP, VE, DE, PI, MN, ...
}

\author{
Åsa Larson ${ }^{1, a}$, Sifiso Nkambule ${ }^{1}$, Emelie Ertan ${ }^{1}$, Josefine Söder $^{1}$ and Ann E. Orel ${ }^{2, b}$ \\ ${ }^{1}$ Dept. of Physics, Stockholm University, 10691 Stockholm, Sweden \\ ${ }^{2}$ Dept. of Chemical Engineering and Materials Science, University of California, Davis, USA
}

\begin{abstract}
The resonant states of $\mathrm{HeH}$ are computed by combining structure calculations at a full configuration interaction level with electron scattering calculations carried out using the Complex-Kohn variational method. We obtain the potential energy curves, autoionization widths, as well as non-adiabatic couplings among the resonant states. Using the non-adiabatic couplings, the adiabatic to diabatic transformation matrix can be obtained. A strict diabatization of the resonant states will be used to study various scattering processes where the resonant states are involved. These processes involve high energy dissociative recombination (DR) and ion-pair formation (RIP), resonant and direct dissociative excitation (DE), penning ionization (PI) as well as mutual neutralization (MN).
\end{abstract}

\section{Introduction}

When an electron collides with a molecular ion, such as $\mathrm{HeH}^{+}$, a number of processes can occur. There is, of course, elastic scattering

$$
\mathrm{HeH}^{+}\left(v_{i}, J_{i}\right)+\mathrm{e}^{-} \rightarrow \mathrm{HeH}^{+}\left(v_{i}, J_{i}\right)+\mathrm{e}^{-},
$$

where only the momentum of the electron has changed. There are inelastic processes, such as vibrational and rotational excitation, where some energy is exchanged

$$
\operatorname{HeH}^{+}\left(v_{i}, J_{i}\right)+\mathrm{e}^{-} \rightarrow \operatorname{HeH}^{+}\left(v_{f}, J_{f}\right)+\mathrm{e}^{-} .
$$

At higher energies, electron-impact excitation to a dissociative electronic state can result in direct dissociative excitation (direct DE). In this case, the incident electron causes a transition to a dissociative excited state of the target ion with enough internal energy to cause fragmentation [1-3]

$$
\mathrm{HeH}^{+}\left(v_{i}, J_{i}\right)+\mathrm{e}^{-} \rightarrow \mathrm{He}^{+}+\mathrm{H}+\mathrm{e}^{-} .
$$

In addition, there are a number of resonant processes. Every state of the ion can serve as a parent ion for a series of neutral states. Below the ground state, these are a Rydberg series of neutral states converging

\footnotetext{
${ }^{\text {a } W o r k ~ s u p p o r t e d ~ b y ~ t h e ~ S w e d i s h ~ R e s e a r c h ~ C o u n c i l ; ~ e-m a i l: ~ a a s a l @ f y s i k . s u . s e ~}$

$\mathrm{b}$ This work is supported by the National Science Foundation, PHY-11-60611, while working at the Foundation. Any opinion, finding and conclusions or recommendations expressed in this material are those of the author and do not necessarily reflect the views of the National Science Foundation; e-mail: aeorel@ucdavis .edu
}

This is an Open Access article distributed under the terms of the Creative Commons Attribution License 4.0, which permits unrestricted use, distribution, and reproduction in any medium, provided the original work is properly cited. 
to the ion. Electron capture to these Rydberg states via non-adiabatic interactions drives the dissociative recombination (DR) process at low collision energies [4-7]

$$
\mathrm{HeH}^{+}\left(v_{i}, J_{i}\right)+\mathrm{e}^{-} \rightarrow \mathrm{He}+\mathrm{H},
$$

where the $\mathrm{H}$ fragment becomes excited.

However, if the parent state is an excited state of the ion, the neutral Rydberg states are doubly excited (Feshbach) resonances that lie below the direct dissociation threshold, and are formed when an incoming electron excites the target ion and attaches to a Rydberg orbital. Capture into these states can initiate a number of resonant processes. As the neutral molecule evolves in time, the system can autoionize, meaning the electron can be re-emitted, returning the molecule to its original electronic state. If the system returns to a bound ro-vibrational state of the ion, the process is resonant vibrational excitation (resonant VE) (or de-excitation)

$$
\operatorname{HeH}^{+}\left(v_{i}, J_{i}\right)+\mathrm{e}^{-} \rightarrow \mathrm{HeH}^{*} \rightarrow \mathrm{HeH}^{+}\left(v_{f}, J_{f}\right)+\mathrm{e}^{-} .
$$

If the system has enough energy to dissociate to products, the process provides an efficient path to dissociation at energies below the direct excitation level and the reaction is resonant dissociative excitation (resonant DE) [3, 8]

$$
\mathrm{HeH}^{+}\left(v_{i}, J_{i}\right)+\mathrm{e}^{-} \rightarrow \mathrm{HeH}^{*} \rightarrow \mathrm{He}+\mathrm{H}^{+}+\mathrm{e}^{-} .
$$

If, while evolving in time, the neutral fragments reach an internuclear separation beyond which autoionization is no longer possible, the states are considered electronically bound and the result is again dissociative recombination

$$
\mathrm{HeH}^{+}\left(v_{i}, J_{i}\right)+\mathrm{e}^{-} \rightarrow \mathrm{HeH}^{*} \rightarrow \mathrm{He}+\mathrm{H} .
$$

The resonant states of $\mathrm{HeH}$ produce a peak in the DR cross section around $18 \mathrm{eV}[9,10]$ and in this process the He fragment becomes excited. At collision energies above $12.07 \mathrm{eV}$ (with target ions in rovibrational groundstate), the energy of system is enough for dissociation into the ion-pair. The resonant ion-pair formation (RIP)

$$
\mathrm{HeH}^{+}\left(v_{i}, J_{i}\right)+\mathrm{e}^{-} \rightarrow \mathrm{HeH}^{*} \rightarrow \mathrm{He}^{+}+\mathrm{H}^{-}
$$

is a processes competing with the high-energy DR [10]. The study of these processes requires both the accurate treatment of the electron scattering processes, but must also include a consistent description of the neutral electronic states for all internuclear distances that are probed during the processes. This includes not only the adiabatic potential energy curves of the resonant states, but also the couplings. Both the couplings between the resonant states and the scattering continuum (the autoionization widths) and the non-adiabatic couplings among the resonant states are needed to completely describe the processes and to compute cross section including the branching ratios into final states.

The same curves and couplings mediate another series of collision processes. These include Penning Ionization (PI) [11-14]

$$
\mathrm{He}^{*}+\mathrm{H} \rightarrow \mathrm{HeH}^{*} \rightarrow \mathrm{He}+\mathrm{H}^{+}+\mathrm{e}^{-} .
$$

Associative Ionization (AI) $[15,16]$

$$
\mathrm{He}^{*}+\mathrm{H} \rightarrow \mathrm{HeH}^{*} \rightarrow \mathrm{HeH}^{+}\left(v_{f}, J_{f}\right)+\mathrm{e}^{-},
$$

and Mutual Neutralization (MN) [17-19]

$$
\mathrm{He}^{+}+\mathrm{H}^{-} \rightarrow \mathrm{HeH}^{*} \rightarrow \mathrm{He}^{*}+\mathrm{H} .
$$

Our work in this area has been to develop methods to obtain these curves and couplings and next we will use them to study the above mentioned processes. In the next section we will outline our computational 
approach both for the quantum chemistry calculations of the adiabatic potential energy curves and non-adiabatic couplings as well as the electron scattering calculations. This is followed by a section describing the diabatization procedure needed. We will then discuss our methods that will be applied to study some of the above mentioned processes and some preliminary results are presented.

\section{Computational approach}

The goal is to obtain an accurate and consistent set of curves and couplings that can be used in the study of these processes. Our computational approach to this problem combines quantum chemistry and electron scattering techniques carried out with the MESA program [20]. We use standard quantum chemistry methods to obtain the bound electronic states and the non-adiabatic coupling between those states. We then carry out electron scattering calculations to obtain the positions of the resonant states and the autoionization widths. It is important that these calculations are done accurately and consistently to obtain the potential energy curves and couplings. When the potential energies and autoionization widths of the resonant states as well as the non-adiabatic interactions between them are obtained, a strict diabatization procedure is followed.

\subsection{Structure calculations}

For $\mathrm{HeH}$, there are two distinct sets of neutral electronic states. First there are the low lying electronic states that can be described as Rydberg states with an ionic core in the electronic ground state $\left(X^{1} \Sigma^{+}\right)$. These states drive the low-energy dissociative recombination of $\mathrm{HeH}^{+}$through non-adiabatic interactions. The second set of states are higher lying electronic states that are the Rydberg states with an excited (either $a^{3} \Sigma^{+}$or $A^{1} \Sigma^{+}$) ionic cores. These resonant states are important for high-energy dissociative recombination, resonant pair formation resonant dissociative and vibrational excitation, penning ionization and mutual neutralization. The present article focuses on these states.

To obtain the bound adiabatic potential energy curves, we carried out a full Configuration Interaction (CI) calculation with an aug-cc-pVQZ He basis [21] set and an aug-cc-pVTZ basis set for H [22]. Extra diffuse functions were added to describe the $3 \mathrm{~d}$ states. This resulted in 106 basis functions. This reproduced the asymptotic limits of resonant states to $\sim 200 \mathrm{~cm}^{-1}$ with an accuracy for the relative energies of $\sim 5 \mathrm{~cm}^{-1}$.

Below the ion, all states have configurations of $(1 \sigma)^{2}(n \lambda)^{1}$, which are a series of Rydberg states converging to the ground state of the ion and are bound electronically. In Fig. 1, the potential energies of the ground state $\left(X^{1} \Sigma^{+}\right)$of the $\mathrm{HeH}^{+}$ion and the first two excited states $\left(a^{3} \Sigma^{+}\right.$and $\left.A^{1} \Sigma^{+}\right)$are displayed with the thick black curves. The bound Rydberg states of ${ }^{2} \Sigma^{+}$and ${ }^{2} \Pi$ symmetries are shown with the thin dotted black and red curves, respectively.

Above the ion, states with configurations $(1 \sigma)^{2}(n \lambda)^{1}$, are background states, representing a discretized ionization continuum. However, there exists electronic states with configurations $(1 \sigma)^{1}(2 \sigma)^{1}(n \lambda)^{1}$ that are members of Rydberg series converging to excited states of the ion (either $a^{3} \Sigma^{+}$or $A^{1} \Sigma^{+}$). Eleven resonant states of ${ }^{2} \Sigma^{+}$symmetry, six states of ${ }^{2} \Pi$ symmetry and two ${ }^{2} \Delta$ states were obtained. The potential energies of these states can be extracted from the structure calculation by examining the configurations of the wave functions. As long as the autoionization width of the resonant state is small and there is no strong mixing of the resonant and background configurations, the structure calculations can provide reliable energy positions of the resonant states. In Fig. 1 the HeH resonant states of ${ }^{2} \Sigma^{+}$(thin black curves), ${ }^{2} \Pi$ (thin red curves) and ${ }^{2} \Delta$ (thin green curves) are displayed.

We first focus on the resonant states of ${ }^{2} \Sigma^{+}$symmetry. One of these states asymptotically correlates with the ion-pair $\mathrm{He}^{+}+\mathrm{H}^{-}$limit. When the attractive ion-pair state interacts with the covalent Rydberg states avoided crossings occur at large internuclear distances. The eleven resonant states of ${ }^{2} \Sigma^{+}$ symmetry include all resonant states with avoided crossings at distances smaller than $100 \mathrm{a}_{0}$. The 


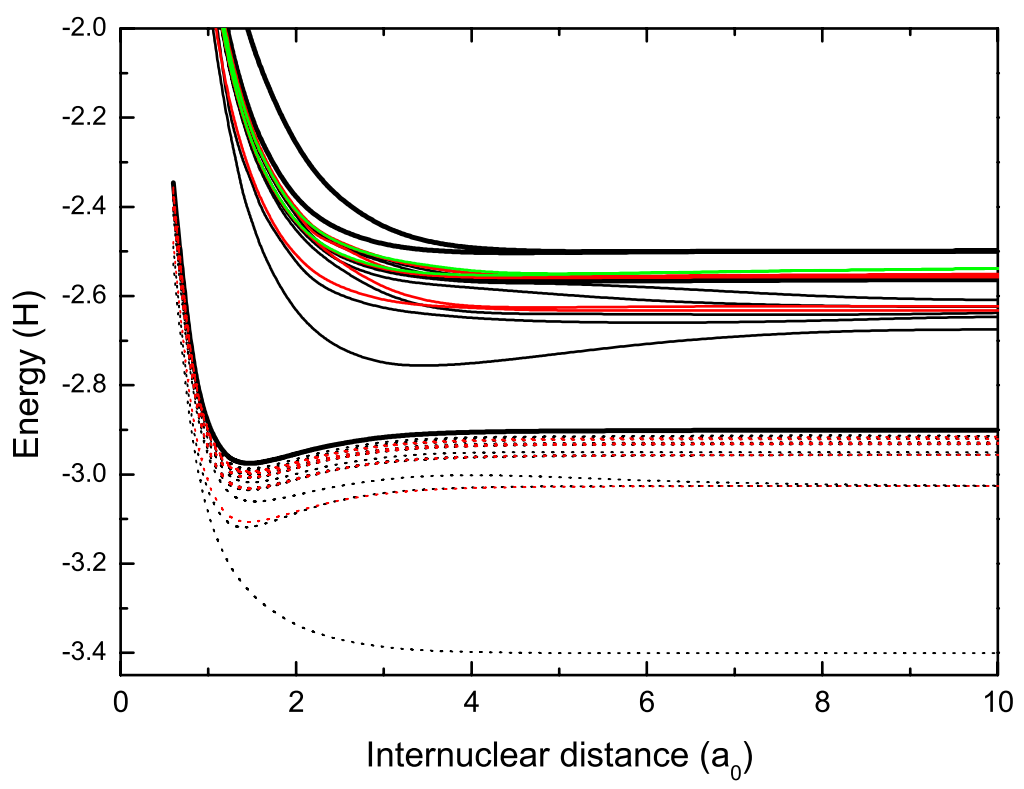

Figure 1. Thick black curves show the potential energies of the ground and first two excited states of $\mathrm{HeH}^{+}$. The thin dotted curves show bound Rydberg states, while thin solid curves are the potential energies of the resonant states of ${ }^{2} \Sigma^{+}$symmetry (black), ${ }^{2} \Pi$ symmetry (red) and ${ }^{2} \Delta$ symmetry (green).

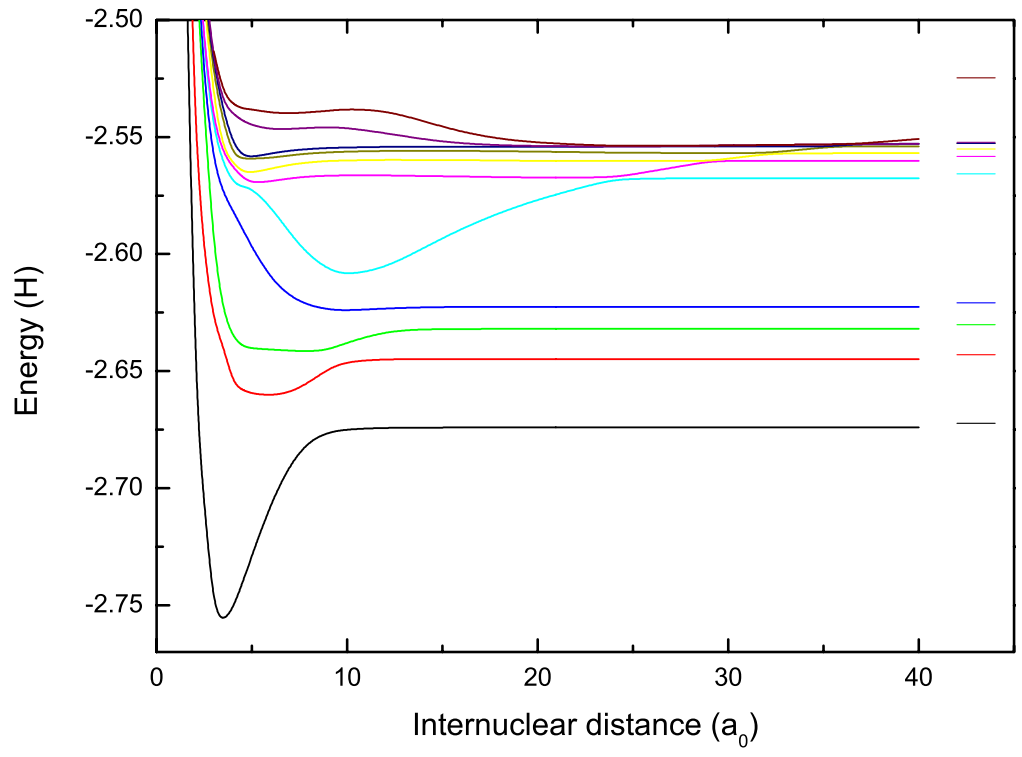

Figure 2. Potential energies of $\mathrm{HeH}$ resonant states of ${ }^{2} \Sigma^{+}$symmetry.

potential energies of the adiabatic resonant states of ${ }^{2} \Sigma^{+}$symmetry are displayed in Fig. 2 . The thin lines at $R>40 \mathrm{a}_{0}$ show experimental dissociation limits relative to the ground state fragments. There are clear signs of avoided crossings occurring when the attractive ion-pair state interact with the Rydberg states at large distances. For Rydberg states dissociating into the $\operatorname{He}(n=2)+H$, these avoided crossings occur 


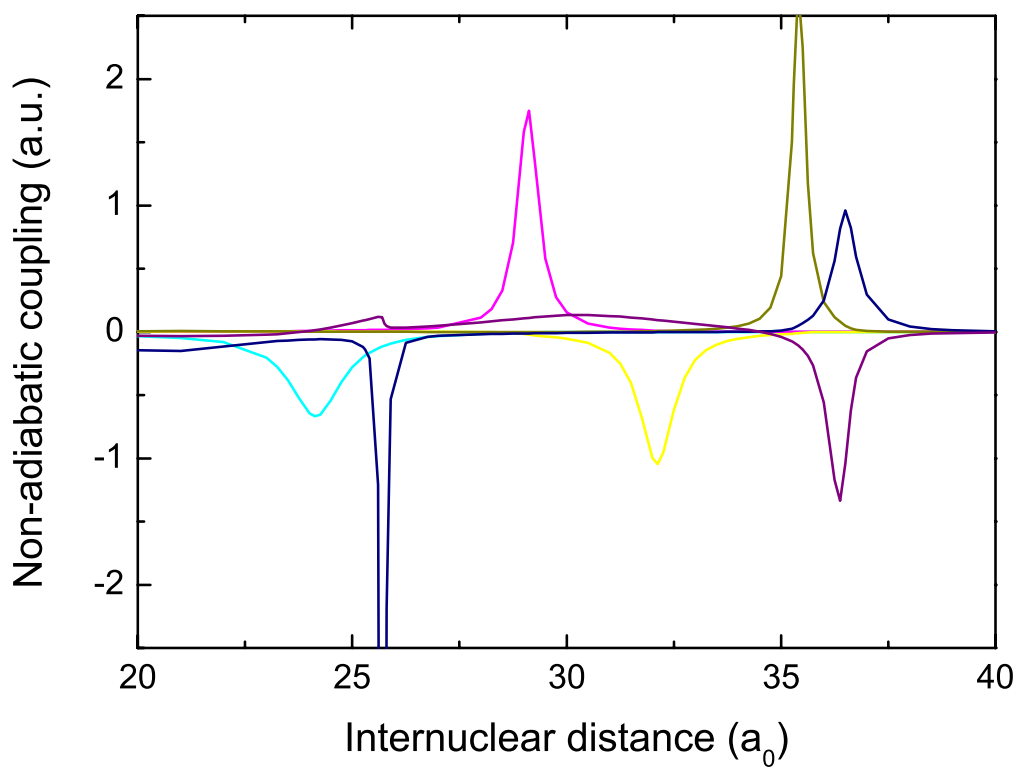

Figure 3. Non-adiabatic coupling elements between neighboring adiabatic states involved in the $\mathrm{He}(n=3)+\mathrm{H}-$ ion pair avoided crossings.

around $10 \mathrm{a}_{0}$. For the $\operatorname{He}(n=3)+\mathrm{H}$ states there are avoided crossings ranging from 24 to $37 \mathrm{a}_{0}$. The avoided crossings are accurate to within a few tenths of $\mathrm{a}_{0}$.

The non-adiabatic couplings, $f_{i j}(R)=\left\langle\Phi_{i}^{a d}\left|\frac{\partial}{\partial R}\right| \Phi_{j}^{a d}\right\rangle$, were calculated analytically using the MESA code. These calculations could not be carried out at the full-CI level. They employed the same basis, but a Multi-Configuration Self Consistent Field (MCSCF) calculation was used to generate the orbitals. An active space of ten natural orbitals was used, with full CI in the active space and double external excitations. This resulted in the same energies as the full CI calculation. The resonance states were identified by configurations and the couplings between them were computed. The approach is straightforward at large internuclear distances where the autoionization widths of the resonant states are negligible. However, at small distances $\left(R<5 \mathrm{a}_{0}\right)$ there is strong mixing between the ionization continuum and the resonant states. Work is presently in progress to extract coupling elements when each resonant state can be described as a superposition of roots from the structure calculation.

There are arbitrary signs of the adiabatic electronic wavefunctions. To correct for this sign-flipping, a minimization program is written where the signs of the states are determined by minimizing the sum of the differences of all coupling elements at neighboring grid points. Our preliminary results of some of the coupling elements involved in the $\mathrm{He}(n=3)+\mathrm{H}$ and $\mathrm{He}^{+}+\mathrm{H}^{-}$avoided crossings are shown in Fig. 3. The couplings are peaked at the vicinity of the avoided crossings.

\subsection{Electron scattering calculations}

The electron scattering calculations could not be carried out at the full-CI level. These calculations, using the complex Kohn variational method [23], employed the same basis. Natural orbitals were obtained a full-CI level. An active space of ten natural orbitals was used, with full $\mathrm{CI}$ in the active space and single external excitations. By fitting the eigenphase sum of the fixed nuclei elastic scattering matrix to a BreitWigner form, the resonance energy position (relative to the target ion) and the autoionization width can 

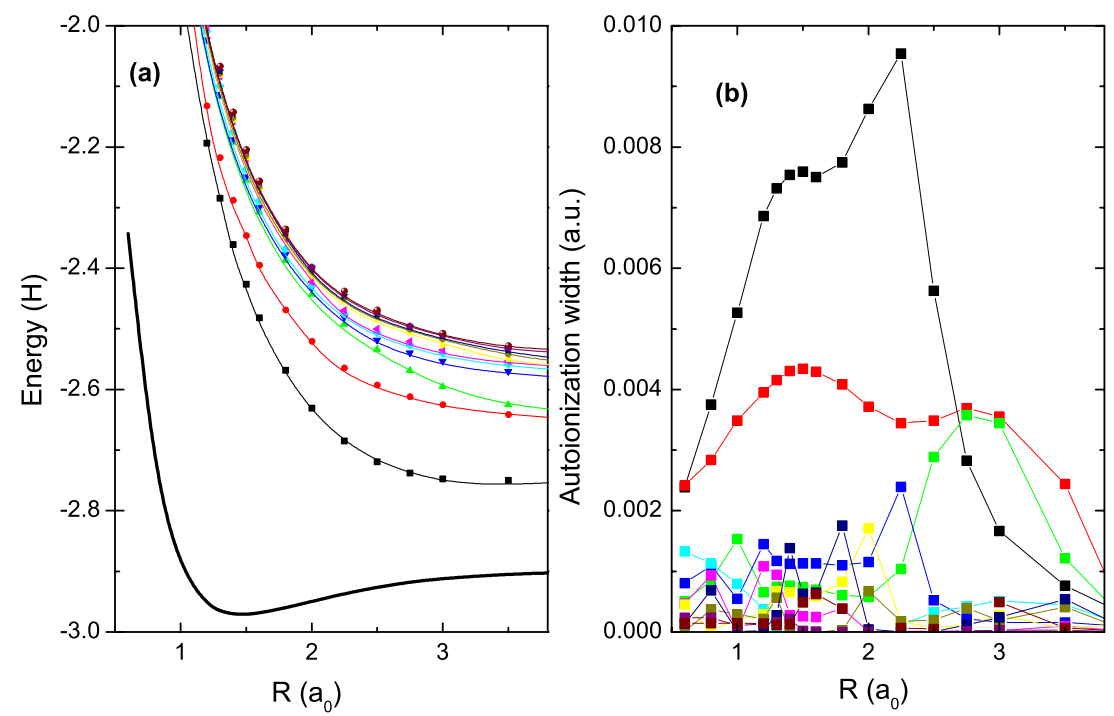

Figure 4. a) Comparison of the energies of the ${ }^{2} \Sigma^{+}$resonant states obtained from structure calculations (lines) and electron scattering calculations (symbols). b) Autoionization widths of the ${ }^{2} \Sigma^{+}$resonant states obtained from electron scattering calculations.

be obtained. This resulted in shifted energies, but when referenced relative to the ion, resulted in the same results. The results are shown in Fig. 4.

\subsection{Diabatization procedure}

We include 11 resonant states of ${ }^{2} \Sigma^{+}$symmetry. We assume these states interact with the ionization continuum [through the autoionization width $\Gamma_{i}$ ], and they interact with each other [through the non-adiabatic couplings $f_{i j}(R)$ ]. We do not include interactions to higher lying resonant states or between states of different symmetry. Usually the transformation between the adiabatic and diabatic representations are approximate. In the case of a diatomic, where the curves and non-adiabatic couplings are known, it is possible to use the exact "strict diabatization". Further information can be found in paper [24]. In this case, the adiabatic resonant states can be transformed to a diabatic representation using the orthogonal transformation matrix $\mathbf{T}$ obtained by integrating the equation [24]

$$
\left[\mathbf{1} \frac{d}{d R}+\mathbf{f}\right] \mathbf{T}=\mathbf{0},
$$

where the off-diagonal anti-symmetric f-matrix contains the non-adiabatic coupling elements. We apply the boundary condition that asymptotically the adiabatic and diabatic states are identical. Once the transformation matrix is obtained, the diagonal complex adiabatic potential matrix $\mathbf{V}^{a d}$ with elements $V_{i i}^{a d}(R)=V_{i}(R)-i \frac{\Gamma_{i}(R)}{2}$, can be transformed to a diabatic potential matrix $\mathbf{V}^{d}=\mathbf{T}^{t} \mathbf{V}^{a d} \mathbf{T}$. We use a local complex model for the autoionization [25], which is known to be valid for high-energy resonant states. The resulting real part of the diagonal elements of the diabatic potential matrix are displayed in Fig. 5. It should be noted that using a "strict diabatic" representation, the diagonal potentials do not remind of the more familiar shape of the "quasi-diabatic potentials" with an attractive ion-pair potential crossing the repulsive Rydberg states. 


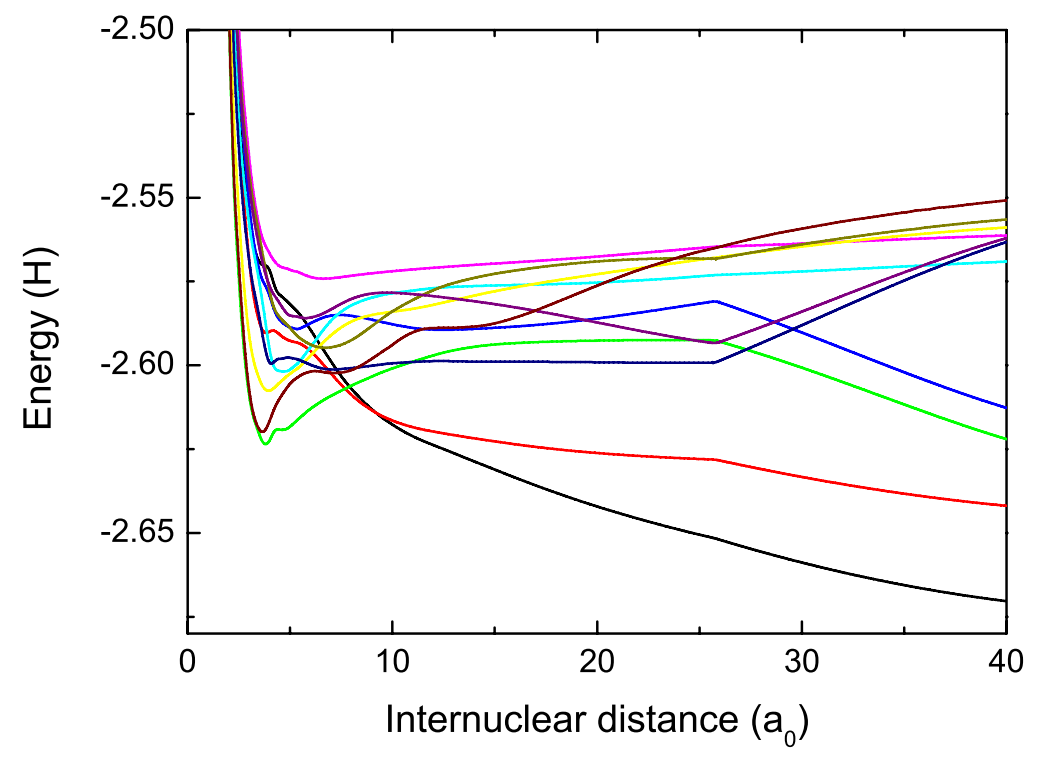

Figure 5. Real part of the diagonal elements of the diabatic potential matrix in ${ }^{2} \Sigma^{+}$symmetry.

\subsection{Nuclear dynamics}

Once the potential energy curves, couplings and autoionization widths of the $\mathrm{HeH}$ resonant states are determined, the reactions discussed in the introduction can be studied. The nuclear dynamics are studied quantum mechanically by numerically solving either the time-dependent [26] or time-independent driven Schrödinger equation [27]. For some processes, such as dissociative recombination, ion-pair formation and dissociative excitation, the rotational motion of the system will be neglected. Studies of reactions involving collisions of atoms or ions (such as penning ionization, associative ionization and mutual neutralization) require inclusion of angular momentum. A partial wave decomposition is performed and the coupled radial Schrödinger equation will be solved for fixed angular momenta [28]. The total cross section for the reaction is obtained by summarizing contributions from partial waves.

\section{Results}

We report some preliminary results from the studies of direct and resonant dissociative excitation processes. These calculations can be seen as a test of the accuracy of the electron scattering calculations upon which the present model is based.

\subsection{Direct dissociative excitation}

In direct dissociative excitation, inelastic electron scattering causes an excitation of the $\mathrm{HeH}^{+}$ion to one of the lowest two excited states $\left(a^{3} \Sigma^{+}\right.$or $\left.A^{1} \Sigma^{+}\right)$, followed by a direct dissociation to $\mathrm{He}^{+}+\mathrm{H}$. Within the adiabatic-nuclei approximation [29, 30], the energy-normalized nuclear continuum function of the excited target state is approximated with a Dirac-delta function. Then the cross section can be written $[2,31]$

$$
\sigma_{n n^{\prime}}^{\Lambda}(E)=\int_{R_{E}}^{\infty} \tilde{\sigma}_{n n^{\prime}}^{\Lambda}(E, R)\left[\chi_{v_{0}}(R)\right]^{2} d R,
$$




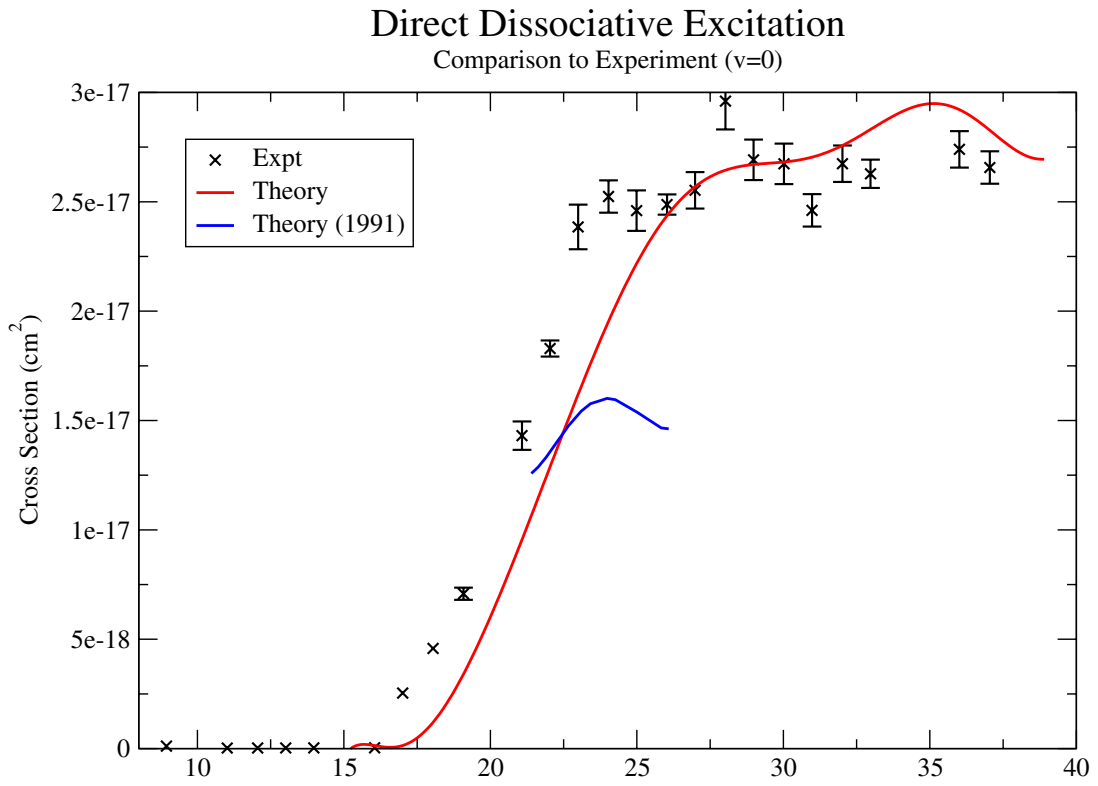

Figure 6. Cross section for direct dissociative excitation of $\mathrm{HeH}^{+}$.

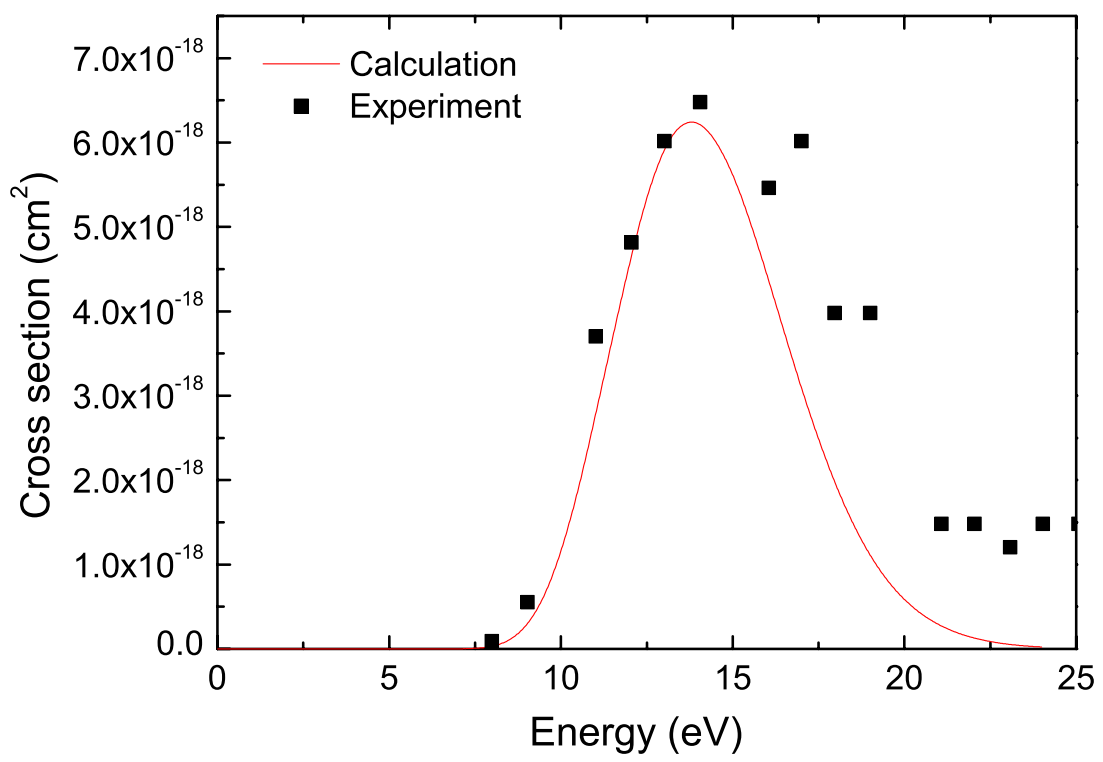

Figure 7. Cross section for resonant dissociative excitation of $\mathrm{HeH}^{+}$.

where the fixed-nuclei inelastic electron scattering cross section, $\tilde{\sigma}_{n n^{\prime}}^{A}(E, R)$ obtained from the ComplexKohn calculations, is averaged over the square of the vibrational wave function of the $\mathrm{HeH}^{+}$ion in the initial vibrational state $v_{0}$. The fixed-nuclei inelastic cross section has sharp, narrow resonances on a smooth and relative energy-independent background. The sharp resonances arise from capture into Feshbach resonances and to obtain only the direct contribution these were manually removed. In Fig. 6, the calculated cross section (red curve) is compared with the measured cross section using an ion-storage 
ring [3]. We also compare with the computed cross section (blue curve) by Orel and Kulander [2] that was based on the same delta-function approximation, but with a smaller electron scattering calculation and assuming that the fixed nuclei cross section simply shifts with the threshold energies when the internuclear distance is changed. We have begun to explore methods that go beyond the delta-function approximation and that include the nuclear dynamics in the description of the process [31].

\subsection{Resonant dissociative excitation}

In the resonant dissociative excitation process, the electron is temporarily captured into the resonant states. Autoionization back to the ground ionic state may cause dissociation if the kinetic energy of the $\mathrm{He}+\mathrm{H}^{+}$fragments is high enough. As a model to test the scattering calculation, we have only included the contribution from the lowest resonant state of ${ }^{2} \Sigma^{+}$symmetry. We do not include non-adiabatic couplings to higher resonant states. As can be seen in Fig. 7, the computed cross section [32] agrees well with the measured cross section from CRYRING [3]. At higher collision energies $(E>14 \mathrm{eV})$, higher lying resonant states contribute to the cross section. Next, the process will be studied by including all resonant states as well as the couplings between them.

\section{Conclusion}

We have carried out combined structure/electron scattering calculations on $\mathrm{HeH}$ to obtain accurate energies, non-adiabatic couplings as well as autoionization widths of resonant states. These electronic states are important for many reactive collisional processes such as high-energy dissociative recombination, ion-pair formation, vibrational and dissociative excitation, associative ionization, penning ionization as well as mutual neutralization. Using a strict diabatic representation of the resonant states, consistent studies of these processes will be performed.

\section{References}

[1] F. B. Yousif and J. B. A. Mitchell, Phys. Rev. A, 40 (1989) 4318

[2] A. E. Orel, T. N. Rescigno, and B. H. Lengsfield III, Phys. Rev. A 44 (1991) 4328

[3] C. Strömholm, J. Semaniak, S. Rosén, H. Danared, S. Datz, W. van der Zande, and M. Larsson, Phys. Rev. A 54 (1996) 3086

[4] S. L. Guberman, Phys. Rev. A 49 (1994) R4277

[5] B. K. Sarpal, J. Tennyson, and L. A. Morgan, J. Phys. B: At. Mol. Opt. Phys. 27 (1994) 5943

[6] H. Takagi, Phys. Rev. A 70 (2004) 022709

[7] A. Larson and A. E. Orel, Phys. Rev. A 72 (2005) 032701

[8] A. E. Orel and K. C. Kulander, Phys. Rev. A 54 (1996) 4992

[9] G. Sundström, S. Datz, J. R. Mowat, S. Mannervik, L. Broström, M. Carlsson, H. Danared, and M. Larsson, Phys. Rev. A 50 (1994) R2806

[10] Å. Larson and A. E. Orel, Phys. Rev. A 59 (1999) 3601

[11] K. L. Bell, J. Phys. B: At. Mol. Opt. Phys. 3 (1970) 1308

[12] A. P. Hickman and H. Morgner, J. Chem. Phys. 67 (1977) 5484

[13] H. Waibel, M. -W. Ruf, and H. Hotop, Z. Phys. D 9 (1988) 191

[14] A. Merz, M. W. Muller, M. -W. Ruf, H. Hotop, W. Meyer, and M. Movre, Chem. Phys. 145 (1990) 219

[15] G. D. Magnuson and R. H. Neynaber, J Chem. Phys. 60 (1974) 3385

[16] D. Nehari, F. Brouillard, D. Jureta, and X. Urbain, J. Phys. B: At. Mol. Opt. Phys. 35 (2002) 4733

[17] B. Peart and D. A. Hayton, J. Phys. B: At. Mol. Opt. Phys. 27 (1994) 2551 
[18] K. Olamba, S. Szücs, J. P. Chenu, Naji El Arbi and F. Brouillard, J. Phys. B: At. Mol. Opt. Phys. 29 (1996) 2837

[19] M. Chibisov, F. Brouillard, J. P. Chenu, M. H. Cherkani, D. Fussen, K. Olamba, and S. Szücs, J. Phys. B: At. Mol. Opt. Phys. 30 (1997) 991

[20] MESA (Molecular Electronic Structure Applications) (1990) P. Saxe, B. H. Lengsfield, R. Martin and M. Page

[21] D. E. Woon and T. H. Dunning Jr., J. Chem. Phys. 100 (1994) 2975

[22] T. H. Dunning Jr., J. Chem. Phys. 90(1989) 1007

[23] T. N. Rescigno, C. W. McCurdy, A. E. Orel, and B. H. Lengsfield III, The Complex Kohn Variational Method in Computational methods for electron-molecule scattering eds. Huo W H and Gianturco F A (Plenum New York) (1995)

[24] C. A. Maed and D. G. Truhlar, J. Chem. Phys. 77 (1982) 6090

[25] C. W. McCurdy and J. L. Turner, J. Chem. Phys. 78 (1983) 6773

[26] J. B. Roos, A. E. Orel and A. Larson, Phys Rev. A 79 (2009) 062510

[27] J. B. Roos, A. Larson and A. E. Orel, Phys Rev. A 78 (2008) 022508

[28] M. Stenrup, Å. Larson and N. Elander, Phys. Rev. A 79 (2009) 012713

[29] D. M. Chase, Phys. Rev. 104 (1971) 838

[30] M. Shugard and A. U. Hazi, Phys Rev. A 12 (1975) 1895

[31] E. Ertan A time-dependent description of dissociative excitation of $\mathrm{HeH}^{+}$Master thesis in computational physics, Dept. of Physics, Stockholm University, Sweden, 2013

[32] J. Söder, Resonant dissociativ excitation av $\mathrm{HeH}^{+}$Bachelor thesis in physics, Dept. of Physics, Stockholm University, Sweden, 2013 